\title{
Medical Image Compression using Adaptive Subband Threshold
}

\begin{abstract}
Vidhya $\mathbf{K}^{\dagger}$
Abstract - Medical imaging techniques such as Magnetic Resonance Imaging (MRI), Computed Tomography (CT) and Ultrasound (US) produce a large amount of digital medical images. Hence, compression of digital images becomes essential and is very much desired in medical applications to solve both storage and transmission problems. But at the same time, an efficient image compression scheme that reduces the size of medical images without sacrificing diagnostic information is required. This paper proposes a novel threshold-based medical image compression algorithm to reduce the size of the medical image without degradation in the diagnostic information. This algorithm discusses a novel type of thresholding to maximize Compression Ratio (CR) without sacrificing diagnostic information. The compression algorithm is designed to get image with high optimum compression efficiency and also with high fidelity, especially for Peak Signal to Noise Ratio (PSNR) greater than or equal to $36 \mathrm{~dB}$. This value of PSNR is chosen because it has been suggested by previous researchers that medical images, if have PSNR from $30 \mathrm{~dB}$ to $50 \mathrm{~dB}$, will retain diagnostic information. The compression algorithm utilizes one-level wavelet decomposition with threshold-based coefficient selection.
\end{abstract}

Keywords: Discrete wavelet transform, Medical imaging, Image compression, Entropy coder, Performance measures

\section{Introduction}

In medical applications, compression method aims to reduce the size of the image without degradation in the diagnostic information. Thus, the development of efficient compression scheme is an ongoing challenge in medical fields, as loss of information may mislead diagnosis.

The image compression schemes can be classified into two categories, namely: Lossless image compression and Lossy image compression. In lossless image compression, the reconstructed image is numerically and visually identical to the original image. The level of image compression achieved can be represented by Compression Ratio (CR). The CR achieved for lossless techniques are typically around 2:1 to 3:1 [1]. In lossy image compression, higher $\mathrm{CR}$ can be achieved when compared to lossless compression techniques but the reconstructed image contains degradations relative to the original image. A lossy compression method is called visually lossless when the loss of information caused by compression method is invisible for an observer.

Image compression can be accomplished by the use of coding methods, spatial domain techniques and transform domain techniques. Spatial domain methods which operate directly on the pixels of the image combine spatial domain algorithms and coding methods. In transform domain technique, image transforms are used to decorrelate the pixels and the information packed coefficients are

$\dagger$ Corresponding Author: Dept. of Electronics and Communication Engineering, Vel Tech, Chennai, India. (kvidhyasri@gmail.com) Received: November 13, 2014; Accepted: September 28, 2015 quantized and coded. Discrete Cosine Transform (DCT) and Discrete Wavelet Transform (DWT) are the most popular transform techniques [2, 3]. Huffman coding and Arithmetic coding are the most popular entropy coding techniques $[3,4]$.

The well-known DCT based Joint Photographic Experts Group (JPEG) compression standard divides the image into sub images $[2,3]$. DCT is performed on the sub-images, and the resulting coefficients are quantized and coded. The main disadvantage of DCT is that at low bit rates, it yields blocking artifacts.

The image compression methods that use wavelet transforms have been successful in providing high rates of compression while maintaining good image quality and are superior to DCT-based methods [5-7].

After the introduction of DWT, a wide variety of wavelet-based compression methods [5] have been reported. Among them, Embedded Zero tree Wavelet (EZW) which uses parent-child dependencies between subband coefficients at the same spatial location of wavelet decomposed image, Set Partitioning in Hierarchical Trees (SPIHT) which uses the self similarity between subbands of wavelet decomposed image and JPEG2000 algorithm [8, 9] which uses Embedded Block Coding with Optimized Truncation (EBCOT) are the most popular methods.

In teleradiology applications, several research works have been performed to determine the degree of compression that maintains the diagnostic image quality [10]. Sone et al. proved that there is no loss in diagnostic accuracy for compression ratios up to 9:1 [11]. Compression of medical images is vital in achieving a low bit rate in the 
representation of radiology images in order to reduce the data volume, without loss in diagnostic information [12, 13]. Persons et al. discussed diagnostic accuracy and reported that reconstructed medical images with a compression ratio of 9:1 do not result in visual degradation [14]. Li et al. investigated the effect of JPEG and wavelet compression algorithm on medical images and concluded that compression ratio up to 10:1 is acceptable [15].

Asraf et al. proposed a compression technique, which is a hybrid of lossless and lossy techniques using neural network vector quantization and Huffman coding [16]. This high complexity technique is tested for medical images achieving compression ratio of 5 to 10 . Chen proposed a new algorithm for medical image compression that is based on SPIHT algorithm [1]. An $8 \times 8$ DCT approach is adopted to perform subband decomposition. SPIHT is then employed to organize data. This is blockbased technique and more complexity is involved. The author concluded that for application in medical domain some adaptations are needed to remove blocking artifacts.

All these techniques discussed involve multiple levels of wavelet decomposition resulting in high computational complexity. Higher level of decomposition results in more details that can be thresholded to get high compression ratios but leads to energy loss. Energy retained will be more if the image is decomposed to fewer levels but compression attained is less [17]. The issue is not whether to compress medical images using lossless or lossy techniques, but preferably which type of compression can be used without compromising image quality [18].

This paper presents a novel type of thresholding to maximize CR without sacrificing diagnostic information.

This paper is organized as follows. Section 2 presents the proposed method. Section 3 presents experimental results. Section 4 presents conclusions.

\section{Materials and Methods}

The medical images are decomposed using one-level Daubechies 9/7 wavelet filter into low-frequency components (approximation subband LL) and high frequency components (detail subbands LH, HL and HH). This wavelet is chosen because it is superior in providing reconstructed images with high quality due to high energy compaction $[19,20]$. The proposed medical image compression with thresholding (MICT) algorithm is developed with one level wavelet decomposition in the image.

To achieve compression, optimum number of significant coefficients is to be selected. This is performed in the proposed compression technique by a novel method of thresholding.

The compression algorithm, when applied to medical images should not affect the visual quality and hence the diagnostic information. This is achieved in this method by considering the Peak Signal to Noise Ratio (PSNR) as the quality parameter for the compression. PSNR which relates Mean Square Error (MSE) to the maximum amplitude of the original image is calculated as given in Equation (1), by comparing original image $\mathrm{x}(\mathrm{i}, \mathrm{j})$ of size $\mathrm{M} \times \mathrm{N}$ pixels with reconstructed image $x_{R}(i, j)$ of size $M \times N$ pixels.

$$
P S N R \text { in } d B=10 \log _{10}\left(\frac{255^{2}}{M S E}\right)
$$

where

$$
M S E=\frac{1}{M x N} \sum_{i} \sum_{j}\left(x(i, j)-x_{R}(i, j)\right)^{2}
$$

In image compression, acceptable values of PSNR are in the range of $30 \mathrm{~dB}$ and $50 \mathrm{~dB}$ where higher is better [21, 22]. Russakoff et al. proved that while comparing reconstructed images with original image, a few visible differences could be noticed for PSNR above $30 \mathrm{~dB}$ [23]. So the work is proposed for yielding best visual quality images with PSNR equal to or greater than $36 \mathrm{~dB}$, as there is no visual degradations in images reconstructed with PSNR greater than $36 \mathrm{~dB}$ [23-25].

\subsection{Methodology}

\subsubsection{One level wavelet decomposition}

The original image is decomposed using Daubechies 9/7 wavelet. The DWT of the image is calculated by passing it through a series of digital filters namely low pass filter and high pass filter. The wavelet transform decomposes the image into different frequency subbands. The low pass filter extracts coarse information of the signal and the high pass filter extracts the detail information of the signal. The image is filtered along the row and decimated by two followed by filtering the sub-image along the column and decimated by two which results in four frequency subbands, namely LL, LH, HL and HH respectively. The proposed MICT technique uses the approximation subband coefficients and detail subband coefficients obtained in the one level decomposition of DWT.

\subsubsection{Adaptive subband threshold}

The proposed MICT employs adaptive subband threshold i.e. different threshold for the four subbands. If all subbands are processed with single threshold, then there is a possibility of losing the diagnostic information of the image. The threshold for selection of coefficients obtained in the first level of decomposition is computed based on the information content of the image for achieving compression without any observable loss of information. The threshold is determined by examining the number of occurrences of each coefficient as described below. The number of occurrences of each coefficient in subband is 
initialized to one. When a coefficient is read, the count of that particular coefficient is incremented. The coefficient with maximum count is identified and that coefficient is chosen as initial threshold value. The reason for choosing the coefficient which occurs more number of times is because it contributes more to the image. This process is applied independently on each subband. At each iteration, the procedure of threshold selection is applied on the left over coefficients of the corresponding subbands.

\subsubsection{Proposed MICT}

For processing the approximation subband LL, the threshold is determined by examining the occurrences of each coefficient in that particular subband. Initially the threshold is the most frequently occurring approximation coefficient, the contribution of which is high in the decomposed image. The approximation subband coefficients are selected by testing their significance by comparing with the determined threshold. Then the approximation subband coefficients that have a magnitude greater than or equal to the threshold are identified as significant coefficients. The same procedure is applied independently to identify the significant coefficients from the detail subbands LH, HL and $\mathrm{HH}$ by comparing with the threshold, which is the coefficient with maximum occurrence in each of the detail subbands. LL, LH, HL and HH coefficients greater than or equal to their respective thresholds are significant coefficients. The remaining coefficients of the subbands are retained. This condition is chosen to select the minimal and optimum number of coefficients to achieve compression. This procedure is adopted because the same threshold cannot be used for all the subbands as the information carried or represented by the wavelet coefficients in the subbands differ. As far as medical image is concerned, it is essential to extract the significant information in each of the subbands.

Now, it becomes essential to check whether optimum number of coefficients is selected so that image quality is retained. This is performed as follows. With the chosen significant coefficients by the above procedure, the pixel information is reconstructed by performing inverse DWT (IDWT). Then PSNR is calculated by comparing original image with reconstructed pixel information.

If PSNR is greater than or equal to $36 \mathrm{~dB}$, then the procedure of selection of coefficients is stopped. If the condition is not arrived, the significant coefficients from the remaining coefficients of LL, LH, HL and HH subbands are identified by comparing with their next subsequent threshold i.e. the coefficient with number of occurences lower than the previous threshold and higher than all the remaining ones. The procedure is repeated until the condition of PSNR $\geq 36 \mathrm{~dB}$ is satisfied. Then the significant approximation and detail coefficients are arithmetic coded. In the proposed work, arithmetic coding is used as it provides better compression ratios than Huffman coding
[26].

The flowchart of the proposed MICT encoder showing the identification of the significant approximation and detail subband coefficients is shown in Fig. 1. The original image obtained is decomposed using DWT into approximation coefficients and detail coefficients $(C)$. Initially the zero value significant coefficient matrices (SC and $\mathrm{SC}$ ) of original image size are considered. In approximation subband, the threshold (Th) is the approximation coefficient with maximum frequency of occurrence. The same approach is applied independently to remaining detail subbands. After determining threshold Th of each subband, compare subband coefficients with their respective threshold Th. The coefficients of $\mathrm{C}$ which are greater than or equal to their subband threshold Th are identified and padded to appropriate positions in SC1 matrix. The same approach is applied independently to remaining detail subbands to identify significant coefficients and moved to appropriate positions in SC1 matrix. The remaining coefficients less than threshold Th, are retained in $\mathrm{C}$ matrix itself. Then SC1 having significant coefficients of subbands is added to zero value matrix SC and the result is stored in SC. SC1 is cleared to all zeros. IDWT is performed on SC having

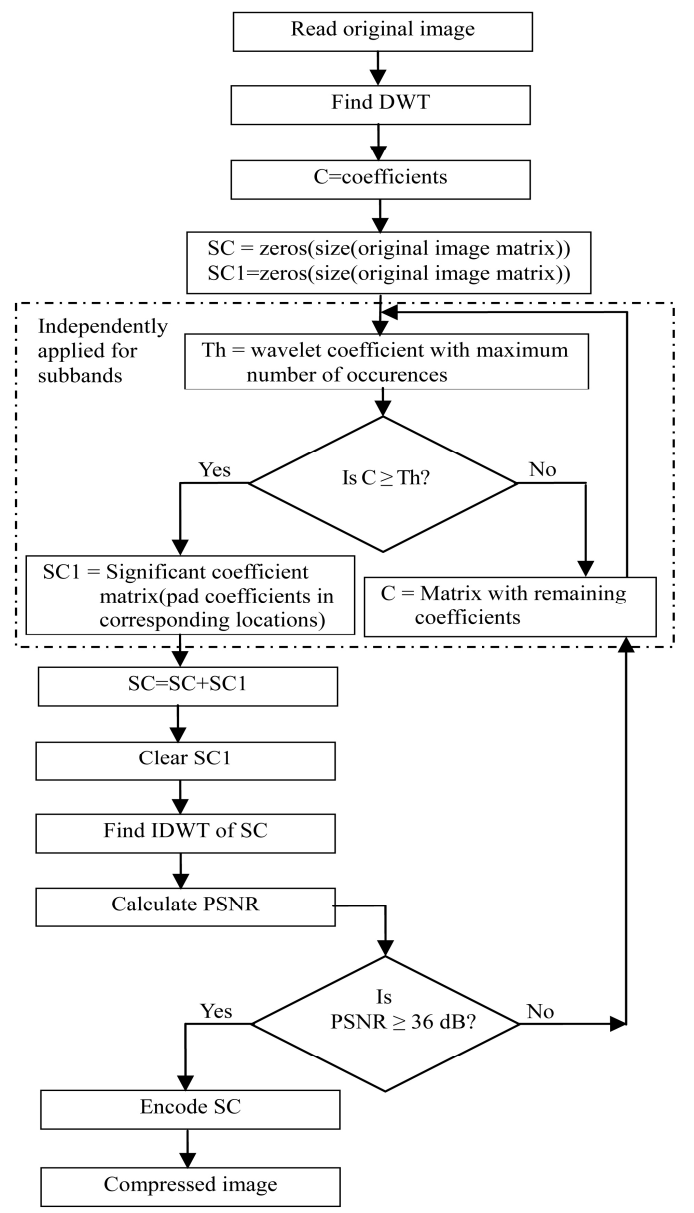

Fig. 1. Flowchart of the proposed MICT algorithm encoder portraying the identification of significant coefficients 
Table 1. Comparison of PSNR, IQI, MSSIM and CR of MICT with JPEG and SPIHT for MRI images

\begin{tabular}{|c|c|c|c|c|c|c|c|c|c|c|}
\hline \multirow{2}{*}{ Images } & \multirow{2}{*}{$\mathrm{CR}$} & \multicolumn{3}{|c|}{ PSNR } & \multicolumn{3}{|c|}{ IQI } & \multicolumn{3}{|c|}{ MSSIM } \\
\hline & & JPEG & SPIHT & MICT & JPEG & SPIHT & MICT & JPEG & SPIHT & MICT \\
\hline MRI image 1 & 3.3 & 4405 & 46.21 & 46.92 & 0.9455 & 0.9217 & 0.9649 & 0.9806 & 0.9826 & 0.9837 \\
\hline MRI image 2 & 3.7 & 42.30 & 43.34 & 46.60 & 0.8831 & 0.8333 & 0.8690 & 0.9636 & 0.9647 & 0.9648 \\
\hline MRI image 3 & 4.2 & 42.14 & 41.66 & 45.27 & 0.7550 & 0.7434 & 0.7964 & 0.9446 & 0.9449 & 0.9443 \\
\hline MRI image 4 & 4.3 & 38.12 & 40.22 & 40.26 & 0.6710 & 0.5540 & 0.6657 & 0.9019 & 0.9054 & 0.8957 \\
\hline MRI image 5 & 4.4 & 44.8 & 46.27 & 48.94 & 0.8626 & 0.8059 & 0.8805 & 0.9741 & 0.9868 & 0.9824 \\
\hline MRI image 6 & 4.7 & 42.63 & 44.64 & 45.45 & 0.7238 & 0.5939 & 0.7622 & 0.9726 & 0.9745 & 0.9751 \\
\hline MRI image 7 & 4.7 & 38.99 & 42.25 & 41.32 & 0.4542 & 0.4627 & 0.9400 & 0.9209 & 0.8839 & 0.9741 \\
\hline MRI image 8 & 4.8 & 42.85 & 44.39 & 44.81 & 0.7687 & 0.6376 & 0.8100 & 0.9738 & 0.9749 & 0.9754 \\
\hline MRI image 9 & 4.8 & 43.58 & 43.87 & 46.89 & 0.6993 & 0.7174 & 0.9506 & 0.9519 & 0.9800 & 0.9773 \\
\hline MRI image 10 & 5.0 & 42.60 & 44.70 & 45.54 & 0.7178 & 0.5858 & 0.7500 & 0.9718 & 0.9775 & 0.9780 \\
\hline MRI image 11 & 5.0 & 43.93 & 44.88 & 46.24 & 0.4864 & 0.5729 & 0.6732 & 0.9397 & 0.9736 & 0.9784 \\
\hline MRI image 12 & 5.0 & 43.24 & 44.76 & 45.49 & 0.7253 & 0.6339 & 0.8154 & 0.9614 & 0.9747 & 0.9752 \\
\hline MRI image 13 & 5.5 & 40.31 & 43.63 & 43.59 & 0.4417 & 0.4576 & 0.9000 & 0.9165 & 0.9111 & 0.9681 \\
\hline MRI image 14 & 5.5 & 44.44 & 44.35 & 47.99 & 0.5353 & 0.5966 & 0.8725 & 0.9381 & 0.9725 & 0.9872 \\
\hline MRI image 15 & 5.7 & 37.93 & 41.89 & 43.00 & 0.6603 & 0.6740 & 0.7160 & 0.9068 & 0.8625 & 0.9780 \\
\hline MRI image 16 & 6.2 & 38.45 & 40.52 & 42.14 & 0.8250 & 0.8470 & 0.9400 & 0.9166 & 0.8583 & 0.9790 \\
\hline MRI image 17 & 6.5 & 36.76 & 40.98 & 42.77 & 0.6702 & 0.6860 & 0.7650 & 0.9154 & 0.8432 & 0.9760 \\
\hline MRI image 18 & 6.5 & 43.50 & 41.05 & 44.85 & 0.4182 & 0.3911 & 0.6900 & 0.9284 & 0.8912 & 0.9785 \\
\hline MRI image 19 & 7.3 & 44.03 & 41.82 & 48.86 & 0.3886 & 0.3900 & 0.8150 & 0.9186 & 0.8912 & 0.9800 \\
\hline MRI image 20 & 8.3 & 40.73 & 40.92 & 45.41 & 0.4003 & 0.4096 & 0.8870 & 0.9117 & 0.8385 & 0.9825 \\
\hline Average & 5.3 & 41.79 & 43.12 & 45.12 & 0.6516 & 0.6257 & 0.8232 & 0.9404 & 0.9296 & 0.9716 \\
\hline
\end{tabular}

significant coefficients to reconstruct the pixel information. Then PSNR is calculated by comparing the original image with reconstructed pixel information. If PSNR is equal to or greater than or equal to $36 \mathrm{~dB}$, the $\mathrm{SC}$ matrix is encoded to construct the compressed image. If a quality criterion is not met, then next Th of each of the subband (which is the coefficient with next highest frequency of occurrence in each of the subband from the remaining coefficients retained in $\mathrm{C}$ to identify significant coefficients from remaining coefficients) is determined and procedure of coefficient selection and quality evaluation is repeated, as illustrated in the flowchart.

The algorithm reconstructs the original image by employing the inverse steps: the compressed image is arithmetic decoded and then IDWT is performed.

\subsection{Algorithm of MICT}

The steps present in the above said proposed MICT algorithm are as follows:

1. Get the input image.

2. Initialize matrices SC and SC1 of size each equal to image size.

3. Decompose the image into approximation subband (low frequency components) and detail subbands (high frequency components) using Daubechies 9/7 wavelet.

4. Identification of significant coefficients

4.1. Determine the threshold (Th) (approximation wavelet coefficient with highest frequency of occurrence).

4.2. Compare the approximation wavelet coefficients $\mathrm{a}(\mathrm{i}, \mathrm{j})$ with Th.

4.3. If the coefficient $a(i, j) \geq T h$, then it is identified as significant coefficient.

4.4. Move them to appropriate positions in SC1.

4.5. Record the remaining coefficients less than Th.

5. Identify the significant coefficients of the detail subbands by applying the same procedure.

6. Create the matrix SC by putting selected coefficients from all subbands in their appropriate positions.

7. Find IDWT to reconstruct the spatial pixel values.

8. Calculate PSNR by comparing original image with reconstructed pixel values.

9. If $P S N R \geq 36 \mathrm{~dB}$,

Go to step 10 to process the significant coefficients. Else,

Repeat the procedure to identify significant coefficients from remaining coefficients with next threshold (Th) (next threshold is the coefficient with next highest frequency of occurrence from the remaining coefficients recorded).

10. Arithmetic encode the significant wavelet coefficients and construct the compressed bitstream.

11. Decode the compressed bitstream.

12. Perform IDWT and reconstruct the original image.

\section{Results and Discussion}

In this section, the proposed MICT algorithm has been discussed for MRI, CT and US images of sizes $256 \times 256$ pixels with 8 bits per pixel. The medical images from various body organs like chest, abdomen and head are considered.

\subsection{Objective image quality measures}

MICT algorithm is applied on 20 images each in MRI, 
CT and US imaging modalities. The CR and PSNR values are computed for these images after applying MICT, SPIHT and JPEG separately.

Apart from comparing PSNR and CR of different images under various imaging modalities, other quality measures that support subjective measures are also computed. Image quality index (IQI) and mean structural similarity index measure (MSSIM) are the popular measures. The image quality index is designed by modeling any image distortion in terms of three factors: loss of correlation, luminance distortion and contrast distortion [27]. Wang et al. developed a measure of structural similarity which compares local patterns of pixel intensities, normalized for luminance and contrast [28]. If the two images are identical, MSSIM value is 1 . The higher the value of the MSSIM index i.e. when MSSIM index approaches 1 , better is the quality of the image.

Fig. 2 presents MRI image compressed with the proposed MICT method, JPEG and SPIHT. The original MRI images and reconstructed images using JPEG, SPIHT and the proposed MICT are shown in Fig. 2 (a) - (d), respectively for $\mathrm{CR}$ of 5.7. The visual quality of the reconstructed images obtained with the proposed MICT is better compared to SPIHT.

Table 1 gives the comparison of MICT with JPEG and SPIHT in terms of CR, PSNR, IQI and MSSIM. The results of the images are tabulated in the order of increasing compression ratios. The objective measures seen

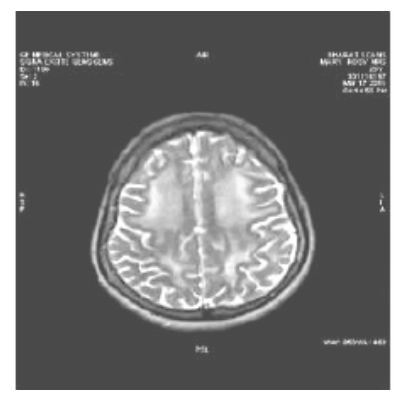

(a)

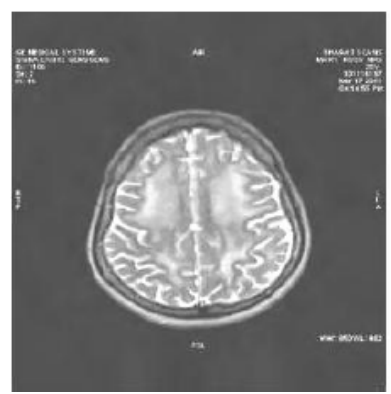

(c)

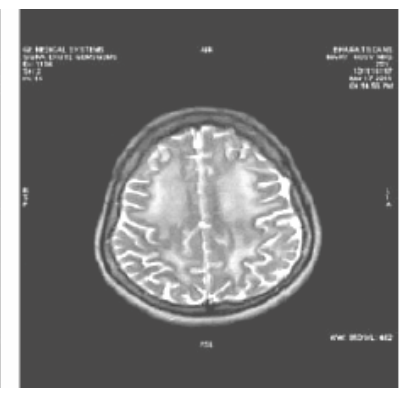

(b)

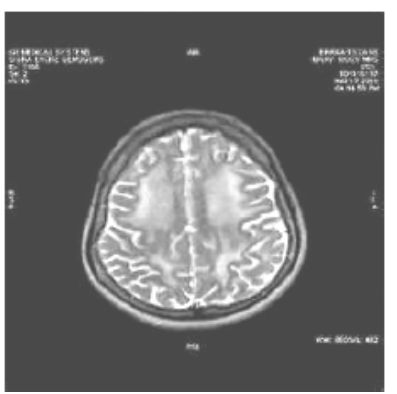

(d)
Fig. 2. (a) Original MRI image (b) Reconstructed image using JPEG (CR=5.7, PSNR=37.93 dB) (c) Reconstructed image using SPIHT $(\mathrm{CR}=5.7$, PSNR $=41.89 \mathrm{~dB})(\mathrm{d})$ Reconstructed image using MICT $(\mathrm{CR}=5.7, \mathrm{PSNR}=43 \mathrm{~dB})$ from Table 1, which compares PSNR and CR for the 20 different MRI images using different compression methods, indicate that the proposed method performs well yielding higher PSNR values compared to other compression methods. The performance of the proposed method is found to be better than JPEG and SPIHT for the same CR. Furthermore, by computing the PSNR average for JPEG, SPIHT and the proposed MICT, the proposed method performs better with an improvement of $3.33 \mathrm{~dB}$ on an average over JPEG and $2 \mathrm{~dB}$ on an average over SPIHT in case of MRI images. The average value of IQI for MICT is 0.8232 which is higher compared to SPIHT with an average of 0.6257 and JPEG with an average of 0.6516 . The higher value of IQI in MICT compared to JPEG and SPIHT can be attributed to the following factor: For an image of given $\mathrm{CR}$, the difference in PSNR between the techniques is less but for the same cases, IQI shows a large difference because in MICT, the coefficients which contain maximum information are collected. In other words, the optimum coefficients which have significant information of the image are selected in MICT technique. This results in better IQI in MICT compared to JPEG and SPIHT, in which blurring of images is a common major problem. The average value of MSSIM for MICT is $0.9716,0.9296$ for SPIHT and 0.9404 for JPEG. All these indicate that MICT achieves equal level of compression with JPEG and SPIHT with higher level of image quality.

Fig. 3 presents CT image compressed with JPEG, SPIHT

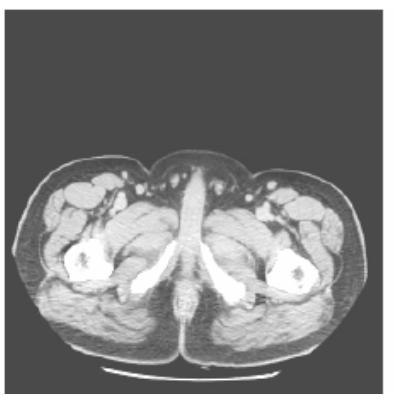

(a)

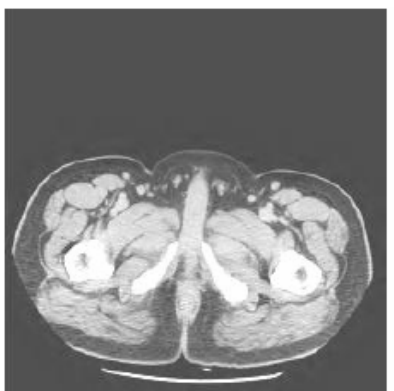

(c)

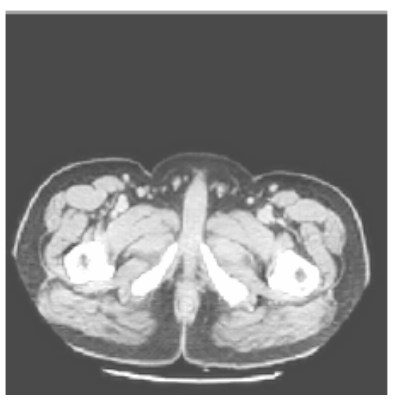

(b)

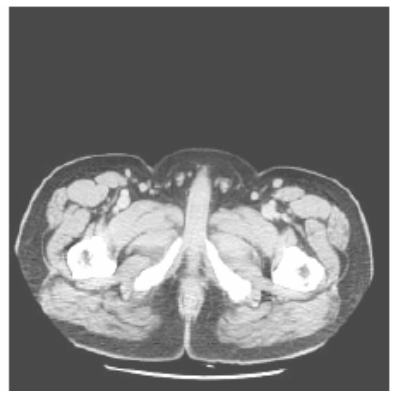

(d)
Fig. 3. (a) Original CT image (b) Reconstructed image using JPEG $(\mathrm{CR}=4.1$, PSNR=38.58 dB) (c) Reconstructed image using SPIHT $(\mathrm{CR}=4.1$, PSNR $=$ $40.78 \mathrm{~dB}$ ) (d) Reconstructed image using MICT $(\mathrm{CR}=4.1, \mathrm{PSNR}=41.21 \mathrm{~dB})$ 
Table 2. Comparison of PSNR, IQI, MSSIM and CR of MICT with JPEG and SPIHT for CT images

\begin{tabular}{|c|c|c|c|c|c|c|c|c|c|c|}
\hline \multirow{2}{*}{ Images } & \multirow{2}{*}{$\mathrm{CR}$} & \multicolumn{3}{|c|}{ PSNR } & \multicolumn{3}{|c|}{ IQI } & \multicolumn{3}{|c|}{ MSSIM } \\
\hline & & JPEG & SPIHT & MICT & JPEG & SPIHT & MICT & JPEG & SPIHT & MICT \\
\hline CT image 1 & 3.4 & 38.05 & 40.19 & 40.79 & 0.6853 & 0.6969 & 0.8400 & 0.9392 & 0.9094 & 0.9423 \\
\hline CT image 2 & 3.6 & 38.63 & 40.41 & 41.77 & 0.6200 & 0.6189 & 0.8531 & 0.9347 & 0.9305 & 0.9533 \\
\hline CT image 3 & 3.7 & 38.52 & 40.68 & 41.42 & 0.6080 & 0.6058 & 0.8538 & 0.9352 & 0.9274 & 0.9536 \\
\hline CT image 4 & 4.1 & 38.58 & 40.78 & 41.21 & 0.5000 & 0.5133 & 0.8944 & 0.9222 & 0.9183 & 0.9539 \\
\hline CT image 5 & 4.2 & 38.91 & 39.76 & 41.39 & 0.5079 & 0.5069 & 0.9082 & 0.9227 & 0.9142 & 0.9593 \\
\hline CT image 6 & 4.3 & 37.25 & 39.80 & 40.74 & 0.4738 & 0.4716 & 0.8315 & 0.9175 & 0.8969 & 0.9618 \\
\hline CT image 7 & 4.3 & 39.20 & 40.19 & 42.42 & 0.4737 & 0.4976 & 0.8870 & 0.9254 & 0.9142 & 0.9594 \\
\hline CT image 8 & 4.5 & 38.91 & 39.76 & 41.39 & 0.5079 & 0.5069 & 0.9082 & 0.9227 & 0.9142 & 0.9593 \\
\hline CT image 9 & 4.6 & 35.17 & 39.21 & 40.16 & 0.4577 & 0.4567 & 0.8600 & 0.9170 & 0.8762 & 0.9632 \\
\hline CT image 10 & 4.6 & 36.57 & 36.73 & 39.72 & 0.4989 & 0.4990 & 0.8346 & 0.9081 & 0.8826 & 0.9448 \\
\hline CT image 11 & 4.7 & 38.39 & 40.09 & 41.29 & 0.4427 & 0.5410 & 0.8845 & 0.9129 & 0.9455 & 0.9496 \\
\hline CT image 12 & 5.0 & 36.17 & 41.00 & 42.88 & 0.5258 & 0.5300 & 0.8986 & 0.9214 & 0.8849 & 0.9665 \\
\hline CT image 13 & 5.2 & 37.00 & 40.62 & 41.45 & 0.4049 & 0.4022 & 0.8164 & 0.9078 & 0.8913 & 0.9561 \\
\hline CT image 14 & 5.2 & 38.46 & 36.75 & 40.99 & 0.5000 & 0.4856 & 0.9000 & 0.9196 & 0.8945 & 0.9547 \\
\hline CT image 15 & 5.3 & 36.22 & 40.77 & 40.95 & 0.4795 & 0.4827 & 0.8536 & 0.9187 & 0.8780 & 0.9559 \\
\hline CT image 16 & 5.5 & 40.20 & 40.89 & 41.75 & 0.7733 & 0.4570 & 0.8318 & 0.9754 & 0.9600 & 0.9703 \\
\hline CT image 17 & 5.5 & 38.00 & 40.25 & 41.40 & 0.3719 & 0.4831 & 0.8514 & 0.9040 & 0.9290 & 0.9467 \\
\hline CT image 18 & 5.5 & 38.64 & 39.09 & 43.00 & 0.5119 & 0.5896 & 0.8784 & 0.9210 & 0.9323 & 0.9624 \\
\hline CT image 19 & 5.8 & 34.36 & 35.28 & 39.26 & 0.4335 & 0.4239 & 0.8100 & 0.9094 & 0.7585 & 0.9596 \\
\hline CT image 20 & 8.7 & 37.15 & 36.47 & 41.38 & 0.3500 & 0.3287 & 0.8230 & 0.8981 & 0.7350 & 0.9642 \\
\hline Average & 4.9 & 37.72 & 39.44 & 41.27 & 0.5063 & 0.5049 & 0.8609 & 0.9216 & 0.8946 & 0.9568 \\
\hline
\end{tabular}

and the proposed MICT method. Fig. 3(a)-(d) shows original CT image and reconstructed images obtained with JPEG, SPIHT and the proposed MICT method, respectively for CR of 4.1 .

Table 2 shows the results of CR, PSNR, IQI and MSSIM obtained for the 20 different CT images with JPEG, SPIHT and the proposed MICT for the same CR. The numerical results seen from Table 2 indicate that the proposed method yields better results compared to other compression methods. The performance of the proposed method has been found to be better than JPEG and SPIHT for the same CR. Moreover, taking the PSNR average for JPEG, SPIHT and the proposed MICT, the proposed method gives performance of $3.55 \mathrm{~dB}$ higher PSNR than that of JPEG and $1.83 \mathrm{~dB}$ higher PSNR than SPIHT, in case of CT images. MICT has an average IQI of 0.8609 compared to 0.5063 for JPEG and 0.5049 for SPIHT. In the same way, the average MSSIM for MICT is high with a value of 0.9568 compared to 0.9216 for JPEG and 0.8946 for SPIHT. These results again show that in CT images also, for identical CR, MICT preserves higher information compared to JPEG and SPIHT.

Fig. 4 presents US image compressed with different compression algorithms. Fig. 4 (a)-(c) show the original US image and reconstructed images obtained with JPEG, SPIHT and the proposed MICT, respectively for CR of 4.4.

The tabular results of CR, PSNR, IQI and MSSIM seen from Table 3 compare the proposed method with SPIHT and JPEG, compression method for the 20 different US images. By computing the PSNR average for JPEG, SPIHT and the proposed MICT, the performance of the proposed method has been found to be better than SPIHT and JPEG for the same CR. The proposed method gave a performance of $4.56 \mathrm{~dB}$ higher PSNR than JPEG and $1.06 \mathrm{~dB}$ higher

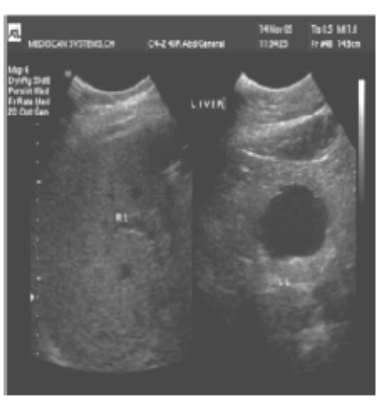

(a)

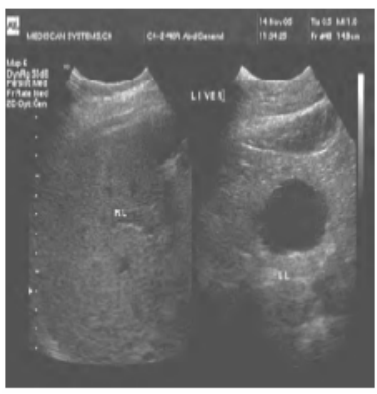

(c)

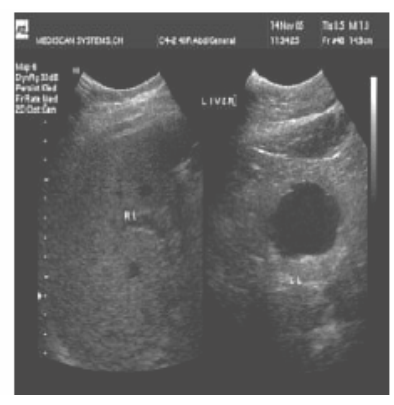

(b)

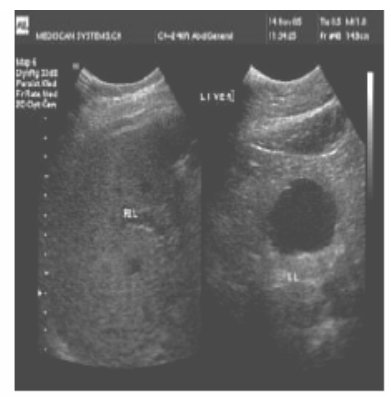

(d)
Fig. 4. (a) Original US image (b) Reconstructed image using JPEG $(\mathrm{CR}=4.4, \mathrm{PSNR}=37.64 \mathrm{~dB})$ (c) Reconstructed image using SPIHT $(\mathrm{CR}=4.4$, PSNR $=$ $38.97 \mathrm{~dB}$ ) (d) Reconstructed image using MICT $(\mathrm{CR}=4.4, \mathrm{PSNR}=41.04 \mathrm{~dB})$

PSNR than SPIHT. The average value of IQI for MICT is 0.8390 which is higher compared to SPIHT with an average of 0.6097 and JPEG with an average of 0.6055 . The reason for this high difference in IQI between MICT and SPIHT is as discussed for MRI images. Similarly the average value of MSSIM for MICT is $0.9484,0.9268$ for 
Table 3. Comparison of PSNR, IQI, MSSIM and CR of MICT with JPEG and SPIHT for US images

\begin{tabular}{|c|c|c|c|c|c|c|c|c|c|c|}
\hline \multirow{2}{*}{ Images } & \multirow{2}{*}{$\mathrm{CR}$} & \multicolumn{3}{|c|}{ PSNR } & \multicolumn{3}{|c|}{ IQI } & \multicolumn{3}{|c|}{ MSSIM } \\
\hline & & JPEG & SPIHT & MICT & JPEG & $\begin{array}{l}\text { SPIHT } \\
\end{array}$ & MICT & JPEG & SPIHT & MICT \\
\hline US image 1 & 3.8 & 36.24 & 39.17 & 40.41 & 0.6798 & 0.6795 & 0.8557 & 0.9368 & 0.9376 & 0.9384 \\
\hline US image 2 & 3.9 & 33.86 & 38.98 & 39.67 & 0.6295 & 0.6359 & 0.8142 & 09338. & 0.9235 & 0.945 \\
\hline US image 3 & 4.0 & 36.45 & 38.83 & 40.36 & 0.6514 & 0.7197 & 0.8325 & 0.9358 & 0.9190 & 0.9405 \\
\hline US image 4 & 4.0 & 36.36 & 38.87 & 40.14 & 0.6684 & 0.6798 & 0.8641 & 0.9253 & 0.9264 & 0.9314 \\
\hline US image 5 & 4.0 & 34.57 & 40.80 & 40.66 & 0.5203 & 0.5718 & 0.8248 & 0.9232 & 0.9362 & 0.9512 \\
\hline US image 6 & 4.0 & 36.68 & 37.22 & 41.44 & 0.7046 & 0.8062 & 0.8368 & 0.9472 & 0.9408 & 0.9556 \\
\hline US image 7 & 4.3 & 34.77 & 40.08 & 40.13 & 0.5060 & 0.5400 & 0.8661 & 0.9188 & 0.9403 & 0.9515 \\
\hline US image 8 & 4.4 & 37.64 & 38.97 & 41.04 & 0.6506 & 0.6671 & 0.8322 & 0.9241 & 0.9239 & 0.9298 \\
\hline US image 9 & 4.4 & 35.00 & 40.00 & 40.32 & 0.4428 & 0.4692 & 0.8631 & 0.9125 & 0.9407 & 0.9539 \\
\hline US image 10 & 4.5 & 36.69 & 41.00 & 40.71 & 0.5406 & 0.5300 & 0.8692 & 0.9245 & 0.9460 & 0.9549 \\
\hline US image 11 & 4.6 & 35.98 & 40.30 & 40.80 & 0.7668 & 0.7112 & 0.8612 & 0.9423 & 0.9478 & 0.9490 \\
\hline US image 12 & 4.8 & 36.57 & 38.54 & 40.13 & 0.8205 & 0.7817 & 0.8694 & 0.9503 & 0.9307 & 0.9507 \\
\hline US image 13 & 5.0 & 37.53 & 40.80 & 42.12 & 0.4330 & 0.4418 & 0.7685 & 0.9106 & 0.9094 & 0.9553 \\
\hline US image 14 & 5.2 & 37.50 & 40.93 & 42.25 & 0.4256 & 0.4241 & 0.8727 & 0.9098 & 0.9036 & 0.9417 \\
\hline US image 15 & 5.3 & 34.14 & 39.47 & 40.62 & 0.4373 & 0.5076 & 0.7604 & 0.9155 & 0.9217 & 0.9571 \\
\hline US image 16 & 5.3 & 35.86 & 40.61 & 40.70 & 0.5585 & 0.5598 & 0.7510 & 0.9264 & 0.8872 & 0.9499 \\
\hline US image 17 & 5.6 & 36.15 & 40.29 & 40.77 & 0.7044 & 0.6700 & 0.8464 & 0.9412 & 0.9174 & 0.9534 \\
\hline US image 18 & 5.8 & 37.00 & 40.00 & 41.90 & 0.5204 & 0.5258 & 0.9000 & 0.9260 & 0.9398 & 0.9579 \\
\hline US image 19 & 5.8 & 36.29 & 39.73 & 40.98 & 0.6687 & 0.5986 & 0.8369 & 0.9425 & 0.9415 & 0.9550 \\
\hline US image 20 & 5.9 & 35.29 & 36.00 & 36.76 & 0.7817 & 0.6752 & 0.8544 & 0.8971 & 0.9017 & 0.9468 \\
\hline Average & 4.7 & 36.03 & 39.53 & 40.59 & 0.6055 & 0.6097 & 0.8390 & 0.9272 & 0.9268 & 0.9484 \\
\hline
\end{tabular}

SPIHT and 0.9272 for JPEG. It can thus be inferred that in case of US images also, MICT confirms the preservation of diagnostic information.

MICT has been tested on MRI, CT and US images. The visual quality of reconstructed obtained using the proposed MICT algorithm is better than those obtained using JPEG. Correlation based quality measures MSSIM and IQI give higher values thereby validating that the proposed MICT is better in preserving diagnostic information. Due to the loss of high frequency components, blur can be noticed in edges of the images obtained with JPEG compression method. As JPEG processes compression with 8x8 blocks, it causes blocking artifacts.

In the proposed MICT algorithm, one-level wavelet decomposition is used and hence complexity involved is less. But SPIHT yields better quality images only if level of wavelet decomposition increases. In this work, the reconstructed images using SPIHT is decomposed by 3level wavelet decomposition filter. In terms of subjective quality, resulting images with low level decomposition are almost unusable in SPIHT [29]. So naturally in SPIHT, high complexity is involved since the level of decomposition has to be increased to achieve good image quality. From the images compressed using SPIHT method, edge blur and ringing artifacts are also observed. Ringing artifact is more domineering which causes ripples or oscillations around the edges. The degree of compression attained can possibly be extended by use of many subbands, but leading to a higher complexity in compression methods.

Hence the salient feature of the present MICT work is that it uses one level wavelet decomposition filter. From the results, it is understood that the proposed MICT algorithm yields better quality images compared to SPIHT which causes ringing artifacts and JPEG which causes
Table 4. Average CR and PSNR comparison of MICT with other compression algorithms for different imaging modalities

\begin{tabular}{c|c|c|c|c}
\hline $\begin{array}{c}\text { Imaging } \\
\text { modalities }\end{array}$ & CR & $\begin{array}{c}\text { JPEG } \\
\text { PSNR }(\mathrm{dB})\end{array}$ & $\begin{array}{c}\text { SPIHT } \\
\text { PSNR }(\mathrm{dB})\end{array}$ & $\begin{array}{c}\text { MICT } \\
\text { PSNR }(\mathrm{dB})\end{array}$ \\
\hline MRI images & 5.3 & 41.79 & 43.12 & 45.12 \\
\hline CT images & 4.9 & 37.72 & 39.44 & 41.27 \\
\hline US images & 4.7 & 36.03 & 39.53 & 40.59 \\
\hline
\end{tabular}

blocking artifacts and blur. Another feature of the proposed algorithm is the capability in preserving the vital components of the image which improve the visual fidelity. The developed algorithm yields images with a higher $\mathrm{CR}$ and high fidelity with PSNR $\geq 36 \mathrm{~dB}$, compared to JPEG and SPIHT.

Table 4 summarizes average CR and PSNR comparison of MICT with other coding algorithms for different imaging modalities. From the tabular results, it is clear that the proposed method gives better performance than JPEG and SPIHT in terms of PSNR for the same CR.

\subsection{Subjective image quality analysis}

For subjective evaluation results, the scores of radiologists are involved in this study. The typical subjective method is Mean Opinion Score (MOS). A score of 5 is no distortion (excellent), score of 4 represents a little distortion which can be ignored (good), score of 3 shows that distortion can be seen evidently but it can be accepted (fair), score 2 shows a lot of distortion which cannot be accepted (bad) and score 1 shows too much distortion therefore cannot be tolerated (very bad) [30, 31].Subjective image analysis is the accurate assessment of image quality. A group of observers or experts are involved in the study to assess the 
quality of reconstructed image with respect to the original image. As a part of subjective analysis, clinical validation reports are obtained from radiologists for the proposed work. The clinical validation reports say that most of the radiologists agree with the proposed MICT algorithm and they have given a grade of 4 which indicates that the reconstructed image quality is good.

\section{Conclusion}

MICT algorithm proposed in this paper uses the threshold to detect the coefficients having more influence on the image and is therefore retained. The algorithms differ in the method of identification of significant coefficients. The algorithms work on one level wavelet decomposed image, thresholded and coded with arithmetic coding. Compared to JPEG and SPIHT coding algorithm, the proposed MICT algorithm yields better subjective quality images for the same CR. The performance is evaluated by testing images of various modalities. It has been proved with the help of PSNR, IQI and MSSIM index that more information is preserved for the medical images compressed with the proposed MICT than JPEG and SPIHT. The experimental comparison shows that the proposed MICT algorithm has better performance than other compression standards. The clinical validation reports obtained from the radiologists show the average grading level of 4 which indicates that the image quality reconstructed with the proposed MICT algorithm is good.

\section{References}

[1] Chen Y.Y., "Medical image compression using DCTbased subband decomposition and modified SPIHT data organization," Int. J. Med. Informat., vol. 76, no.10, pp. 717-725, 2007.

[2] Sikora T., "Trends and perspectives in image and video Coding," in Proc. IEEE, vol. 93, no. 1, pp. 617, 2005.

[3] Khalid Sayood, Introduction to data compression, Morgan Kaufmann Publications, 2006.

[4] Singla V., Singla R. and Gupta S., "Data compression modeling: Huffman and Arithmetic," International Journal of The Computer, The Internet and Management, vol. 16, no. 3, pp. 64-68, 2008.

[5] Rehna V.J. and Jeya Kumar M.K., "Wavelet based image coding schemes: A recent survey," International Journal on Soft Computing, vol. 3, no. 3, 2012.

[6] Prabhakar T., Jagan Naveen V., Lakshmi A., Prasanthi G. and Vijaya Santhi G., "Image compression using DCT and wavelet transformations," International Journal of Signal Processing, Image Processing and Pattern Recognition," vol.4, no. 3, 2011.

[7] Rani, B., Bansal, R.K. and Bansal, S. "Comparison of JPEG and SPIHT image compression algorithms using objective quality measures", in Proc. IEEE International Multimedia Signal Processing and Communication Technologies, pp. 90-93, 2009.

[8] Taubman D.S., "High performance scalable image compression with EBCOT," IEEE Trans. Image Process., vol. 9, no. 7, pp. 1158-1170, 2000.

[9] Taubman D.S. and Marcellin M.W., JPEG2000: Ima ge compression fundamentals, standards and practice, Kluwer Academic Publishers, Boston, USA, 2002.

[10] Koff D.A. and Shulman H., "An overview of digital compression of medical images: Can we use lossy image compression in Radiology?," Can. Assoc. Radiol. J., vol. 57, no. 4, pp. 211-217, 2006.

[11] Sone S., Takashima S., Kiyono K., Yang Z.G., Hasegawa M., Kawakami S., Saito A., Hanamura K. and Asakura K., "Effects of JPEG and wavelet compression of spiral low-dose CT images on detection of small lung cancers," Acta Radiol., vol. 42, no. 2, pp. 156-160, 2001.

[12] Chen Y.Y., "Medical image compression using DCTbased subband decomposition and modified SPIHT data organization," Int. J. Med. Informat., vol. 76, no. 10, pp. 717-725, 2007.

[13] Choong M.K., Logeswaran R. and Bister M., "Costeffective handling of digital medical images in the telemedicine environment," Int. J. Med. Informat., vol. 26, no. 9, pp. 646-654, 2007.

[14] Persons K.R., Palisson P.M., Manduca A., Charboneau W.J., James E.M., Charboneau N.T., Hangiandreou N.J. and Erickson B.J., "Ultrasound gray scale image compression with JPEG and wavelet techniques," $J$. Digit. Imag., vol. 13, no. 1, pp. 25-32, 2000.

[15] Li F., Sone S., Takashima S., Kiyono K., Yang Z.G., Hasegawa M., Kawakami S., Saito A., Hanamura K. and Asakura K., "Effects of JPEG and wavelet compression of spiral low-dose CT images on detection of small lung cancers," Acta Radiol., vol. 42, no. 2, pp. 156-160, 2001.

[16] Asraf R., Akbar M. and Jafri N., "Statistical analysis of different image for absolute lossless compression of medical images," in Proc. IEEE Eng. Med. Biol. Soc., pp.4767-4770, 2006.

[17] Tahoces P.G., Varela J.R., Lado M.J. and Souto M., "Image compression: Maxshift ROI encoding options in JPEG2000," Comput. Vis. Image Understand., vol. 109, pp. 139-145, 2008.

[18] Dragan D. and Ivetic D., "A comprehensive quality evaluation system for PACS," UBICC journal, vol. 4, no. 3, pp. 642-650, 2009.

[19] Miaou S.G. and Chen S.T., "Automatic quality control for wavelet based compression of volumetric medical images using distortion constrained adaptive vector quantization," IEEE Trans. Med. Image, vol. 23, no. 11, 2004.

[20] Lin Z., Hoffman M.W., Leon W.D., Schemm N. and 
Balkir S., "A CMOS image sensor with focal plane SPIHT image compression," in Proc. IEEE Int. Symp. on Circuits and Systems, pp. 2134-2137, 2008.

[21] Mastriani M., "Union is strength in lossy image compression," Int. J. Inform. Comm. Eng., vol. 5, no. 2, pp. 102-119, 2009.

[22] Kafri N. and Sulieman H.Y., "Bit-4 of frequency domain-DCT stenography technique," in Proc. IEEE Int. Conf. on Networked Digital Technologies, pp. 286-291, 2009.

[23] Russakoff D.B., Rohlfing T., Mori K. and Rueckert D., Ho A., Adler J.R. and Maurer C.R., "Fast generation of digitally reconstructed radiographs using attenuation fields with application to 2D-3D image registration," IEEE Trans. Med. Imag., vol. 24, no. 11, pp. 1441-1454, 2005.

[24] El Safy R.O., Zayed H.H., El Dessouki A., "An adaptive steganographic technique based on integer wavelet transform," in IEEE Proc., Int. Conf. on Networking and Media Convergence, pp. 111-117, 2009.

[25] Ghasemi E., Shanbehzadeh J. and Fassihi N., "High capacity image steganography using wavelet transform and genetic algorithm," in Proc. Int. Multiconference of Engineers and Computer Scientists, vol. 1, 2011.

[26] Shahbahrami A., Bahrampour R., Rostami M. and Mobarhan M., "Evaluation of Huffman and Arithmetic algorithms for multimedia compression standards," Int. J. Comput. Sci. Eng. Appl., vol. 1, no. 4, pp. 34-47, 2011.

[27] Wang Z. and Bovik A.C., "A Universal image quality index," IEEE Signal Process. Lett., 2002.

[28] Wang Z., Bovik A.C., Sheikh H.R. and Simoncelli, E.P., "Image quality assessment: from error visibility to structural similarity," IEEE Trans. Image Process., vol. 13, no. 4, pp. 600-612, 2004.

[29] Maly J. and Rajmic P., DWT-SPIHT image codec implementation, Brno Univ. of Tech., Crech Republic, 2007.

[30] Saffor A., Ramli A., Ng K. and Dowsett K., "Objective and subjective evaluation of compressed Computed Tomography (CT) images," Internet J. Radiol., vol. 2, no.2, 2002.

[31] Ghrare S.E., Ali M.A.M., Ismail M. and Jumari K., "The effect of image data compression on the clinical information quality of compressed computed tomography images for teleradiology applications," Eur. $J$. Sci. Res., vol. 23, no. 1, pp. 6-12, 2008.

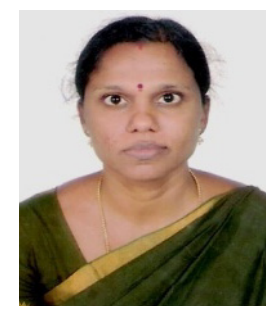

Vidhya $\mathbf{K}$ She received B.E. degree in Electronics and Communication Engineering from Madras University, M.E. degree in Applied Electronics from Madras University and Ph.D. degree in Information and Communication Engineering from Anna University. She has published several articles in refereed journals and conferences. Her research interests include image processing, signal processing and pattern recognition. 